\title{
Sentado en el andén
}

\section{Sitting on the platform}

\author{
Francisco Martínez Sánchez \\ Universidad de Murcia \\ pacomar@um.es
}

Recibido: 05/05/2016

Aceptado: 17/05/2016

Publicado: 30/05/2016

\section{RESUMEN}

En este artículo se realiza una invitación a la reflexión crítica acerca de la evolución de la Tecnología Educativa y del contexto en el que se debería desarrollar su investigación. Una reflexión que se centra sobre todo en el ámbito de la educación superior, la Universidad, como lugar natural para investigar y difundir lo que supone la incorporación de estas tecnologías en la enseñanza y por extensión en la sociedad. Observar desde el andén, como las TIC han logrado situarse como una especialidad en sí mismas creando un distanciamiento de la Tecnología Educativa, dejando tras de sí un empobrecimiento conceptual y de justificación pedagógica de aquellas dentro de la acción didáctica.

\section{PALABRAS CLAVE}

Tecnología Educativa; Universidad; investigación; docencia.

\section{ABSTRACT}

In this article an invitation to critical reflection on the evolution of Educational Technology and the context in which it should develop its research is conducted. A reflection that focuses especially on the field of higher education as a natural place to investigate and disseminate representing the incorporation of these technologies into teaching and by extension in society. Observed from the platform, as ICT have managed to position itself as a specialty, creating a distancing of Educational Technology, leaving behind a impoverishment and pedagogical justification of those within the didactic action.

\section{KEYWORDS}

Educational Technology; University; Research; Teaching.

\section{CITA}

Martínez, F. (2016). Sentado en el andén. RIITE. Revista Interuniversitaria de Investigación en Tecnología Educativa, 0, 17-22. Doi: http://dx.doi.org/10.6018/riite/2016/258131 
Hay dos maneras de ver encenderse la luz en el comienzo y en el final del "tren de Einstein". Los que van dentro la ven de una forma y quienes están en el andén de la estación la ven de otra. Mejor dicho los de dentro, tanto si van al comienzo o al final del tren, ven todos a la vez la luz que se enciende en el centro del mismo. Por el contrario quienes están fuera, en el andén, ven que la luz se enciende en momentos distintos al comienzo que al final del tren.

Este modelo teórico, que nos sirve a los no iniciados para poder entender de alguna manera aspectos de la Teoría de la Relatividad, me sirve para diferenciar entre lo que vemos cuando estamos en el tren y cuando nos hemos bajado de él. Desde el andén, en el que ahora me encuentro, comienzo a ver algunas cosas de manera muy diferente a cuando estaba subido en el tren.

Los medios audiovisuales, la Tecnología Educativa (TE) y ahora las TIC, su función, su integración, su viabilidad, su colaboración a la calidad de la enseñanza, etc. son aspectos que, junto con el vocabulario desarrollado a largo de estos años, deberían de ser ámbitos de reflexión para quienes tienen que ver con este campo del conocimiento, reflexión que ayude a entender, evaluar, eliminar, mejorar y justificar en su caso la situación actual.

Esta reflexión nos debe servir también para evaluar "gurús" que a base de repetir un mismo mantra y que este sea reproducido y amplificado por "acólitos" que, no sé si por incapacidad o por estar "in", parecen no tener ningún tipo de criterio propio sobre aquello que reproducen. Se descubren todos los días mediterráneos a los que, como máxima aportación, se les cambia el nombre 0 , peor aún, se hacen afirmaciones y clasificaciones en base a criterios subjetivos que encierran, en el mejor de los casos, ignorancia. Pero vayamos poco a poco.

Es en la Universidad, entre otras instituciones u organizaciones concretas, donde debe investigarse y difundir lo que supone hoy la incorporación de estas tecnologías en la enseñanza y por extensión en la sociedad. Me centraré en esta ocasión exclusivamente en la enseñanza y en el ámbito universitario.

El quehacer universitario consta, fundamentalmente, de dos aspectos. De un lado la investigación, de otro la docencia. Estas son las dos funciones que tradicionalmente se asigna a los profesores de estas instituciones. Si se acepta este principio, y no hay razones para no hacerlo, tendremos que concluir que la evaluación de estos profesionales deberá contemplar los dos aspectos y más cuando se llega a afirmar, con mucha frecuencia, que en estos centros no se puede hacer una buena docencia si no hay una buena investigación detrás.

Esta última afirmación parece que es la que justifica el tipo de evaluación del profesorado universitario que se está haciendo en este momento, si bien lo realmente importante no es la investigación en sí mismas sino la publicación hecha sobre la misma o sobre cualquier otra cosa y, por encima del contenido, el "dónde" se publicó. La calidad del contenido, la incidencia del mismo en el campo de conocimiento del que hablamos, su repercusión en definitiva, es un aspecto menor. Por este camino se ha llegado a una mercantilización de la difusión del conocimiento, ya que no siempre estas publicaciones contienen mucho conocimiento, pero están en el mercado, en el "centro comercial" adecuado que es lo realmente importante. No importa si fue necesario "comprar" el local.

¿Y cómo se evalúa la docencia? Unas guías didácticas son, en teoría, el referente para esa evaluación, si bien esas guías tienen más una función burocrática que una planificación de la enseñanza. Las guías, una vez publicadas, sirven para tener una base de reclamación del tipo: "no se me aplicó los criterios que hay en ella a la hora de evaluarme ya que el $30 \%$ eran trabajos, el $20 \%$ exposiciones en clase y el $50 \%$ el examen".

La guía didáctica olvida todo lo que tiene que ver con la fase interactiva de la planificación, donde el profesor vaya adaptando el desarrollo de sus clases a la evolución del grupo al que se dirige, sus intereses en cada momento y a la actualidad en el campo de conocimiento concreto. Se suele añadir a estas, una encuesta a los alumnos en la que se pregunta sobre diferentes aspectos de los docentes, pero el resultado de esta encuesta, independientemente de su validez científica, tiene unas repercusiones muy limitadas. 
La relación investigación y docencia, si bien es deseable no por ello debe ser generalizable. No tiene nada que ver la docencia de los primeros años de Universidad con la formación en programas de doctorado o de alta especialización.

En relación con lo dicho incluiré una anécdota personal. Visitando una muy prestigiosa universidad en USA, (la primera o la segunda del ranking mundial) en la que según mi información contaba entre su profesorado con un significativo número de Premios Nobel, comenté al colega que me invitó mí interés en poder saludar a alguno de ellos. Su respuesta fue que estos docentes están con grupos de alumnos muy seleccionados y con un muy alto nivel de formación. Que no daban clase a los grupos de las titulaciones convencionales, y añadió: "en esos niveles podemos dar las clases cualquier profesor".

Un profesor bien preparado y actualizado en su campo de conocimiento está en perfectas condiciones para poder ejercer su docencia en los primeros niveles del currículum universitario, sin necesidad de ser investigador, por lo que su evaluación debe corresponderse con esta función y de manera adecuada.

Los investigadores, que evidentemente han de estar en la Universidad, deben desarrollar su docencia en los niveles donde los resultados de aquella sean más "rentables", donde los alumnos, por su formación, puedan aprovechar los resultados del trabajo investigador del docente. En este caso se deberá evaluar tanto la investigación como la docencia, pero no midiendo el "dónde" se publicó sino "qué se publicó" y "para qué sirvió".

Si bien creo que podría seguir con estas cuestiones, las dejo aquí por el momento, para centrarme en lo que dio origen a este trabajo: la TE. Este campo del conocimiento pedagógico no se salva de lo anteriormente dicho, muy al contrario. A toda la problemática apuntada más arriba hay que añadir la que tiene que ver con su contenido singular, contenido que, junto a unos más o menos teóricos, debe acometer otros de un marcado carácter práctico.

\section{Comenzaré por la docencia.}

La TE tiene la peculiaridad de, entre otras cosas, enseñar a cómo integrar en los procesos de comunicación educativos los diferentes medios, con la idea de mejorar los procesos de enseñanza-aprendizaje, y todo ello ocurre a su vez dentro de un proceso de enseñanza aprendizaje. A esta singularidad hay que unir la permanente evolución de los medios de comunicación y la gran presión económica y social para su incorporación a la enseñanza (véase que digo incorporación que no integración).

Esa presión económico-social a la que me refería, junto a la permanente evolución de las tecnologías, está llevando a un abandono de aquello que da sentido, singularidad y justifica esta materia en la formación de docentes y se va caminando, consciente o inconscientemente, a unos contenidos que dejan de ser propios, entrando en algo que puede contemplarse desde cualquier otro ámbito de la formación docente y que carece de las bases que da sentido a la TE. Me refiero a las TIC, campo surgido de la evolución de la tecnología, que no de la TE, y que, efectivamente puede ser contemplado desde cualquier otra materia. Se deja a un lado los cimientos, aquello que justifica la incorporación de los medios a los procesos de enseñanza y se dedica el tiempo a contemplar la última tecnología sin saber responder pedagógicamente a la eterna pregunta de la TE: ¿por qué este medio aquí y ahora?

A modo de ejemplo en relación a los resultados de la formación de los docentes en el uso de TIC. Existe, al menos un centro de enseñanza secundaria, en el que la pizarra digital se usa al comienzo de clase para recuperar de YouTube un vídeo de yoga que han de atender y seguir todos los alumnos sus indicaciones. Tiene una duración de unos cinco minutos aproximadamente. Todo podría ser más o menos aceptable desde el punto de vista metodológico si la materia a desarrollar en esa clase fuese sobre las culturas orientales o algo por el estilo. El problema radica en que la materia no tiene nada que ver con el yoga y lo que representa a nivel cultural. 
También es conocido el uso de la pizarra digital como pantalla de presentaciones en PowerPoint. Era mucho más económico utilizar la pared. En todo caso la culpa de estas situaciones no es de los docentes.

Para tratar de justificar este cambio se crea un vocabulario nuevo para definir situaciones, modelos, procesos, etc. que ya estaban definidos y cuya denominación estaba aceptada por la comunidad pedagógica. Nuevos nombres para cosas conocidas y nominadas desde antiguo. La única novedad es que ahora están en inglés.

En cuanto a los cambios metodológicos que la incorporación de los nuevos medios debían propiciar se suele quedar en lo anecdótico (hay excepciones muy significativas de carácter muy personal que servirían para confirmar la regla). Muy al contrario las TIC sirven para reforzar los modelos didácticos que se dice se quieren transformar. No puede ser de otra forma cuando se abandonó todo lo que daba sentido y justificaba la incorporación de medios en la enseñanza, la TE en definitiva, habiéndonos quedado sólo con la última tecnología. Es entonces cuando nos quejamos de que "ahora todo el mundo puede formar sobre TIC en la enseñanza" y esto no es que sólo sea un hecho, es una verdad.

Habría que ser coherente y volver a ocuparnos de todo el campo de la TE, de forma que los alumnos adquiriesen una formación adecuada partiendo de la base y, a su vez, quedase claro que se trabaja en un campo diferenciado del resto del abanico curricular de los docentes.

Esta historia comenzó con los medios audiovisuales (MAV) que en su momento tampoco fueron reconocidos como un campo nuevo y singular de formación docente, está identidad sólo se logró cuando se incorporaron una serie de aspectos que daban sentido y justificación al uso de aquellos surgiendo la TE. En ese momento este campo del conocimiento fue reconocido con diferenciado y se integró con identidad propia dentro de los currículums de los docentes. Con las TIC hemos regresado a los MAV prácticamente sin ninguna diferencia.

En este punto recordaría con Burke (2012) en la medida en que hay nuevos conocimientos que se les da entrada en una cultura determinada y se les cede un espacio, por ejemplo en el plan de estudios académicos, algunos de los conocimientos del pasado desaparecen en el transcurso de lo que bien podemos llamar "selección cultural". Esto es lo que ha pasado con la $\mathrm{TE}$, ya que los temas que no están de moda o que se han "enfriado" corren el peligro de ser desechados o, como mínimo, marginados y medio plazo olvidados, quedando siempre al albur de la última ocurrencia. Si bien es necesario una permanente actualización de los conocimientos científicos, ello no debe significar un desconocimiento de lo anterior. Es inevitable una cierta pérdida, aunque pueda ser temporal, de conocimiento como consecuencia de ese avance, ya que como decía Kauffman (2000), "el mero acto de conocer implica un grado de ignorancia”, ignorancia entendida como agnotología.

Con relación a la investigación dentro de este campo ha ocurrido y ocurre una tendencia que impide que se produzcan avances significativos. En este punto hay que hacer un reconocimiento de que existen, de manera minoritaria, investigaciones que han aportado conocimiento para la mejora de situaciones concretas de enseñanza. Cosa diferente es si sus resultados han sido tenidos en consideración por los responsables de las decisiones en política educativa.

No sería capaz de hacer una relación de todas las investigaciones que se han hecho en España sobre, por ejemplo "Actitud y aptitud de los docentes de... ante... (el video, la informática, las TIC)". Todas iguales y todas con los mismos resultados. La justificación es que hay que replicar la investigación dentro de un contexto geográfico concreto, como si la diversidad existente dentro del país fuese tan significativa que justificase la necesidad de un estudio singular.

No entraré en el eterno debate de si investigación básica, denominada así por el ingeniero estadounidense Vannevar Bush o investigación aplicada, tanto una como otra me parecen imprescindibles. En cualquier caso merece la pena destacar con Burke (2012) que la investigación aplicada es un híbrido cultural. No es tanto la derrota del conocimiento académico por el conocimiento práctico como la interpenetración de ambos conocimientos. El problema 
surge cuando sólo se es capaz de hacer una investigación descriptiva sin que esa descripción lleve unida otra investigación que nos facilite estrategias para superar las deficiencias detectadas.

Dejo aquí la investigación relacionada con la TE aunque queda mucho en el teclado. Vayamos al desarrollo de la docencia dentro de la TE.

Hay que partir de las dificultades organizativas y materiales de todo tipo que conlleva cualquier intento de modificación didáctica dentro de la Universidad. Planes de estudio extraños, número de alumnos en las aulas, horarios establecidos en base a criterios más economistas que didácticos, etc. Todo ello hace complicado, cuando no imposibilita, cambios acordes con las posibilidades que las tecnologías pueden aportar a la actualización didáctica que conduzca a una adaptación de las aulas universitarias a la realidad social del entorno y a formar profesionales acordes con las nuevas realidades.

Pero lo dicho no debe conducir a concluir que no es posible hacer nada y que sólo puede hacerse una docencia explicando cómo se hace, cómo se debe hacer, pero no haciendo nada. Sin lugar a dudas la docencia universitaria de TE es el marco ideal para poner en valor y demostrar desde la práctica cómo se puede mejorar utilizando los medios de los que disponemos en este momento.

Por lo general, y como decía más arriba, se está reforzando aquellos modelos pedagógicos que se pretende superar, quedándose en lo anecdótico de los medios o, peor aún en lo espectacular de ellos, sin dar una muestra de lo que se ha de hacer con ellos.

Hoy las TIC han logrado situarse como una especialidad en sí mismas creando un distanciamiento, cuando no un olvido de la TE, con lo que ello supone de empobrecimiento conceptual y de justificación pedagógica de aquellas dentro de la acción didáctica. Finalizo con una cita de Ziman (1995) a la creación de campos de conocimiento excesivamente selectivos: "esta tendencia puede estrechar la mente y dificultar a los estudiosos a tomar incluso su propia disciplina como un todo y olvidar la imagen de conjunto del conocimiento humano" (p. 101).

Termino aquí, sentado en el andén, terminando de ver pasar este tren y esperando al que le sigue, que seguro que viene. Tendrá otra decoración, deberá tener incluso otra forma, otra velocidad, otra máquina, pero lo que sería importante es que fuese un tren que llevase a algún sitio y a su llegada se solucionasen algunos problemas, y en cualquier caso que pudiésemos ver lo mismo los que viajen en él y los que estemos en el andén. En estos momentos veo, como canta Ana Belén: "que a lo lejos silba el viejo tren como sombra del ayer".

\section{REFERENCIAS BIBLIOGRÁFICAS}

Burke, P. (2012). Historia social del conocimiento: de la enciclopedia a wikipedia (II). Paidós.

Kauffman, S. (2000). Investigations. Oxford University Press.

Levie, F. (2006). L'Homme qui voulait classer le monde: Paul Otlet et le mundaneum. Bruselas.

Ziman, J.M. (1995). Of One Mind: The Collectivization of Science. Nueva York: Woodbury.

\section{INFORMACIÓN SOBRE EL AUTOR}

\section{Francisco Martínez Sánchez}

Universidad de Murcia

Actualmente es profesor colaborador en la Universidad de Murcia. Fue profesor Titular de la Universidad de Murcia en el departamento de Didáctica y Organización Escolar desde el año 1986. Antes de ejercer como profesor de esta universidad fue maestro nacional en un centro 
educativo de Murcia y director del C.E.E. Primitiva López. Doctor por la Universidad de Murcia desde el año 1985. Desde entonces se ha dedicado a la docencia e investigación en temas relacionados con la tecnología educativa. Ha dirigido y colaborado diversas investigaciones a nivel internacional, nacional y regional. Desde 1997 es presidente de la Asociación internacional para el desarrollo de la Tecnología Educativa y nuevas tecnologías aplicadas a la educación, conocida como EDUTEC. Ha impartido diversos cursos, talleres, seminarios, etc. a responsables de formación en Argentina, Bolivia, México, Venezuela, Colombia, Cuba, etc., así como en diferentes instituciones educativas españolas.

\section{(cc) EY-NO}

Los textos publicados en esta revista están sujetos a una licencia de Reconocimiento 4.0 España de Creative Commons. Puede copiarlos, distribuirlos, comunicarlos públicamente y hacer obras derivadas siempre que reconozca los créditos de las obras (autoría, nombre de la revista, institución editora) de la manera especificada por los autores o por la revista. La licencia completa se puede consultar en:Licencia Creative Commons Atribución-NoComercial 4.0 Internacional. 